\title{
Design of a Bi-stable Airfoil with Tailored Snap-through Response Using Topology Optimization
}

Bhattacharyya, Anurag; Conlan-Smith, Cian; James, Kai A.

\section{Published in:}

CAD Computer Aided Design

Link to article, DOI:

10.1016/j.cad.2018.11.001

Publication date:

2019

Document Version

Peer reviewed version

Link back to DTU Orbit

Citation (APA):

Bhattacharyya, A., Conlan-Smith, C., \& James, K. A. (2019). Design of a Bi-stable Airfoil with Tailored Snapthrough Response Using Topology Optimization. CAD Computer Aided Design, 108, 42-55.

https://doi.org/10.1016/i.cad.2018.11.001

\section{General rights}

Copyright and moral rights for the publications made accessible in the public portal are retained by the authors and/or other copyright owners and it is a condition of accessing publications that users recognise and abide by the legal requirements associated with these rights.

- Users may download and print one copy of any publication from the public portal for the purpose of private study or research.

- You may not further distribute the material or use it for any profit-making activity or commercial gain

- You may freely distribute the URL identifying the publication in the public portal 


\title{
Design of a bi-stable airfoil with tailored snap-through response using topology optimization
}

\author{
Anurag Bhattacharyya ${ }^{1,}$ \\ University of Illinois Urbana-Champaign, Urbana, IL, 61801 \\ Cian Conlan-Smith ${ }^{1,}$ \\ University of Illinois Urbana-Champaign, Urbana, IL, 61801 \\ Kai A. James ${ }^{1}$, \\ University of Illinois Urbana-Champaign, Urbana, IL, 61801
}

\begin{abstract}
This study aims to harness the geometric non-linearity of structures to design a novel camber morphing mechanism for a bi-stable airfoil using topology optimization. The goal is to use snap-through instabilities to actuate and maintain the shape of the morphing airfoil. Topology optimization has been used to distribute material over the design domain and to tailor the nonlinear response of the baseline structure to achieve the desired bi-stable behavior. The large scale deformation undergone by the structure is modeled using a hyperelastic material model. The non-linear structural equilibrium equations are solved using arc-length and displacement-controlled Newton-Raphson (NR) analysis. Isoparamteric finite element evaluation is used for analyzing kinematic and deformation characteristics of the structure. The optimization problem is solved using a computationally efficient nonlinear optimization algorithm, the Method of Moving Asymptotes (MMA), with a Solid Isotropic Material Penalization (SIMP) scheme. The gradient information required for the optimization has been evaluated using an adjoint sensitivity formulation. Two different design domains, one with a structured quadrilateral mesh and other with an unstructured triangular mesh, are investigated and compared. The effect of different optimization parameters on the final optimized structure and its behavior has also been analyzed. The final result is a novel camber morphing mechanism without the disadvantages of increased weight and higher maintenance costs associated with conventional actuation mechanisms.
\end{abstract}

Keywords: Topology Optimization, Morphing Structures, Bi-stability, Nonlinear elasticity, Unstructured mesh, Triangular finite elements

Email addresses: bhttchr6@illinois .edu (Anurag Bhattacharyya), conlans2@illinois.edu (Cian Conlan-Smith), kaijames@illinois . edu (Kai A. James) 


\section{Introduction}

'Morphing', with respect to aircraft design refers to an aircraft, or by extension, to any part of the aircraft, that is capable of undergoing substantial external shape change which has potential to dramatically alter the aircraft performance to better synchronize with the changing mission environments [1]. Typically, the wings are designed in such a way that it allows the aircraft to fly at a range of flight conditions, but its performance at each of the flight conditions may not be optimal. Fixed wings aircraft are designed to fly optimally only in one flight phase since flying efficiently in multiple flight phases requires dynamic shape change of the wing, in particular, the airfoil camber. The idea is to expand the flight envelope of each aircraft by making it perform optimally in different flight phases. This approach will enable a single aircraft to perform optimally or near-optimally in multiple missions. Morphing has been identified as an effective way to achieve this effect [2]. The shape changes performed in modern-day aircraft are achieved through the movement of flaps and slats, but the performance benefit that could be obtained with fully adaptable and deformable wings may greatly exceed the benefits currently obtained. No matter how one chooses to define 'morphing', there is a general agreement that the conventional hinged surfaces, such as flaps and slats, which move as single entities, cannot be considered 'morphing'. Reich and Sanders [3] listed the major challenges to morphing as: the requirement for the distributed high-power density actuation, structural mechanization, scalability, flexible skins and control law development. Morphing is generally associated with several penalties in terms of cost, complexity and weight. However, in many cases these were overcome with the advantages obtained by morphing. Also, the issues such as unwanted parasite drag, vibrations and noise associated with surface discontinuities (resulting from the movement of flaps and slats as discrete entities) can also be completely eliminated by morphing [4].

During flight, fuel loading and distribution change continuously, especially for HALE (High-Altitude Long Endurance) aircraft, which have a larger portion of fuel weight than any other aircraft. This change in fuel distribution results in large changes to aeroelastic shape throughout the flight, but they are often compelled to fly at non-optimal flight conditions due to air traffic control restrictions. An adaptive wing, whose geometry varies according to changing external aerodynamic load, is capable of optimizing the airflow in each flight regime of the aircraft resulting in an increased aerodynamic performance during cruise. Morphing has been shown to be particularly useful for hunter-killer missions which are mainly cruise and loiter dominated. Roth et al. [5] showed that morphing can have great impact on fleet size for a coast guard patrol mission. The key requirements for these missions are high-altitude cruise with a fast response time, and then a slow, low-altitude control. The absence of sharp edges and deflected surfaces on morphing aircraft provide the potential to reduce its radar signature and visibility, thereby enhancing its stealth properties [6]. The DARPA Mission Adaptive Rotor (MAR) initiative plans to fly an adaptive rotor by 2018. The goal of the project is to develop a rotor that can change its configuration before a mission, in flight between mission segments, and with every revolution. DARPA is looking for morphing solutions which are capable of reducing payload by $30 \%$, increasing range by $40 \%$, reducing the acoustic detection range by $50 \%$, and reducing 
vibration by $90 \%$ over the fixed rotors [1].

Wing morphing can be achieved in three ways: Planform alteration (change of span, chord and sweep), out-of-plane transformation (twist, dihedral/gull and span-wise bending) and airfoil morphing (camber morphing and thickness morphing) [1]. Beaverstock et al. [7] discusses the benefits of camber morphing as compared to span morphing. They observe that, where span morphing requires considerable modification of the planform, camber morphing requires only 5\% trailing edge deflection relative to the cross-sectional chord length. Span morphing can produce up to a $12 \%$ increase in mass before any performance advantage is observed whereas camber morphing causes only up to a $3 \%$ increase. Keeping in mind the benefits that can be derived from the implementation of morphing structures, several authors have investigated different mechanization and actuation techniques to design these structures with improved aerodynamic properties. Woods et al. [8] proposed a biologically inspired morphing concept, FishBAC. It consisted of a flexible skeletal core which was deflected using a system of an antagonistic pair of tendons and a non-backdriveable spooling pulley arrangement. The concept has shown to produce lower drag as compared to the NACA 0012 airfoil with a discrete trailing edge flap. Improvements in lift efficiency were also observed. While the concept is promising and the design was based on the philosophy of simplicity, it still had many individual components which had to function in synchronization to make the wing morph.

Many authors have also investigated the property of bi-stability to design morphing mechanisms. Bi-stable structures have been identified as potential solution in design of morphing mechanisms because of their inherent ability to deform and retain equilibrium in the deformed configuration. Diaconu et al. [9] used a bi-stable laminated composite structure to achieve camber morphing. A bi-stable composite plate was inserted into the airfoil, with the leading edge of the plate fixed to the spar, while the end of the plate was hinged to the airfoil surface to allow for relative rotation of the trailing edge box. Actuation of the bi-stable plate lead to the morphing of the airfoil between two stable states. They were able to achieve the required deflection, but because of the compliant nature of the plates, the structure was not stiff enough to be implemented directly. They suggested the use of stiffeners or additional locking arrangements, which have the potential to increase the weight of the structure and may eventually lead to inefficient performance. Pontecorvo et al. [10] presented a study on bistable arches with a cosine profile, to reduce peak stresses in the arch, designed to be used for morphing applications. They proposed the use of arches as single morphing elements or as elements in multistable morphing honeycomb-like cellular structures. They used arches made of NiTiNOL and Delrin, and studied their force-displacement relation using experimental methods and ANSYS simulations. Their study highlighted the influence of different structural parameters in the design of a bistable arch to meet morphing requirements. Saggere and Kota [11] investigated the use of compliant mechanisms to design a morphing airfoil. They proposed a technique to change the shape of a curved slender rod using a compliant mechanism requiring only a single input actuation force or torque. They used this technique to change shape of leading and trailing airfoil sections.

Taking a different approach many researchers have also investigated the use of smart materials for morphing applications. Smart materials like piezoelectric materials 
(PZT) [12] and Shape Memory Alloys (SMA) [13][14][15] have been extensively explored for designing potential morphing mechanism. The numerous experiments conducted with smart materials indicate that they have the potential to be used for shape control applications, however they still have to overcome numerous challenges before they are finally ready for real world applications. The biggest limitation of smart materials being their low stroke, power and the inverse relation between the strain and operational bandwidth [11].

The current study intends to use snap-through instability, to design a bi-stable airfoil. The choice to use geometric non-linearity for actuation stems from the promising results obtained from numerous studies [16][17][18] that highlight the benefit of using snap-through and buckling instabilities for actuation as opposed to traditional actuators which suffer from issues, such as heavy weight, unreliability, and high maintenance costs. Bi-stability also offers the added advantage of having a self-locking property, as a result, it helps to get rid of redundant and heavy components, increasing system efficiency. Topology optimization has proven to be highly effective in design of structures and mechanisms, as well as materials having unique mechanical and thermal properties [19][20][21][22][23][24]. These design principles can be effectively utilized to design a bi-stable airfoil. The large scale deformation, required for designing structures exhibiting snap-through instability requires the use of non-linear elastic models. Bruns et al.[25] investigated the design of structures exhibiting snap-through by incorporating robust numerical schemes focused on remedying convergence issues. A study by Ramos and Paulino looked into the topology optimization of hyperelasic trusses [26]. James and Waisman [27] focus on design of a bi-stable stent using a hyperelastic material model. The investigators used a neo-hookean hyperelastic method to model the kinematics and deformation characteristics of the solid structure. The non-linear equilibrium equations were solved using a combination of the arc-length method and a displacement-controlled Newton-Raphson procedure.

When it comes to bi-stable structure design, a general trend can be found wherein studies focus on designing structures limited to beams, or designs which can be derived from the classic bi-stable beam design problem. This work is a novel attempt to design a bi-stable morphing airfoil by combining the functionality of bi-stable structures with compliant mechanism design, using topology optimization. There are several advantages to this approach. The design produces a mechanism that requires the lowest actuating energy to change between two stable configurations. Furthermore, no energy is required to maintain the deformed configuration. The structure produced inherently shows multifunctional properties without any increased weight or complexity.

\section{Large Deformation Modeling}

The neo-Hookean material model has been used in this study to characterize the large deformation undergone by structures exhibiting morphing [28][29][30]. The potential energy function is defined using Lamé constants and the right Cauchy-Green 
deformation tensor.

$$
\Phi=\frac{1}{2} \lambda_{0}[\ln J]^{2}-\mu_{0} \ln J+\frac{1}{2} \mu_{0}[\operatorname{tr}(C)-3]
$$

where $J$ is the determinant of the deformation gradient, $\boldsymbol{F}$. The term $\operatorname{tr}(\boldsymbol{C})$ denotes the trace of the right Cauchy-Green deformation tensor. The Second Piola-Kirchoff stress, $\boldsymbol{S}$, can be calculated as twice the derivative of the potential energy function with respect to the right Cauchy-Green deformation tensor.

$$
\boldsymbol{S}=2 \frac{\partial \Phi}{\partial \boldsymbol{C}}=\lambda_{0}[\ln J] \boldsymbol{C}^{-1}+\mu_{0}\left(\boldsymbol{I}-\boldsymbol{C}^{-1}\right)
$$

The Cauchy stress, $\sigma$, is defined as

$$
\boldsymbol{\sigma}=\frac{1}{J} \boldsymbol{F} \boldsymbol{S} \boldsymbol{F}^{\mathrm{T}}
$$

using Equation 2, $\sigma$ can be calculated as

$$
\begin{aligned}
\boldsymbol{\sigma} & =\frac{1}{J}\left[\lambda_{0}[\ln J] \boldsymbol{I}+\mu_{0}\left(\boldsymbol{F} \boldsymbol{F}^{\mathrm{T}}-\boldsymbol{I}\right)\right] \\
& =\frac{1}{J}\left[\lambda_{0}[\ln J] \boldsymbol{I}+\mu_{0}(\boldsymbol{B}-\boldsymbol{I})\right]
\end{aligned}
$$

To keep structural equilibrium, the internal and external forces must be balanced. We can express this condition in residual form as

$$
\boldsymbol{R}=\boldsymbol{f}_{\text {ext }}-\boldsymbol{f}_{\text {int }}=\mathbf{0}
$$

This residual equation gives rise to a nonlinear system of equations which are solved iteratively using a Newton-Raphson procedure to obtain the global vector of nodal displacements, $\boldsymbol{U}$. Dhatt \& Batoz [31], discusses this method in more details. After convergence of Equation 5 , the nodal displacements can be updated by $\Delta \boldsymbol{U}$, where

$$
\boldsymbol{K}_{\text {tan }} \Delta \boldsymbol{U}=\boldsymbol{R}
$$

The matrix $\boldsymbol{K}_{\text {tan }}$ is known as the tangent stiffness matrix. A displacement control method is used to solve the governing equation, $\boldsymbol{R}=0$. The external force used in equation 5 is defined as

$$
f_{e x t}=\theta f_{0}
$$

where $f_{0}$ is a sparse reference vector of size equal to the number of degrees of freedom and a unit value at the degree of freedom corresponding to the prescribed displacement.

\section{Structural Design using Topology Optimization}

\subsection{Design Formulation}

SIMP scheme assignes a design parameter $\rho$ to each element. The effective stiffness of each element, $E_{e}$, is represented as

$$
E_{e}=\rho^{p} E_{0}
$$


where $\rho \in[0,1] . \quad E_{0}$ is the bulk material stiffness of the solid and $p$ is the SIMP penalization parameter. In standard linear elastic problems selecting $\rho_{\min }>0$ prevents the global stiffness matrix from becoming singular.At smaller values of $\rho_{\min }$, the finite element evaluation diverges due to severe mesh distortions and the emergence of degenerate elements. The design of bi-stable mechanisms involves relatively large displacements, and because we are modeling geometric nonlinearity of structures, the algorithm is susceptible to divergence. This forces us to use a higher value of $\rho_{\min }$ to stabilize the algorithm.

Use of lower-order finite elements and a discrete density distribution throughout the design domain can cause numerical instabilities leading to mesh-dependent designs and appearance of checkerboard patterns [32][33]. In order to get rid of these issues we implement a density filtering technique introduced by Bruns and Tortorelli [19]. The set of elemental density variables, $\rho$, is defined as:

$$
\rho=W x
$$

Where the matrix $\boldsymbol{W}$, consisting of normalized weight coefficients, $\tilde{w_{j i}}$, defined as:

$$
\tilde{w_{j i}}=\frac{w_{i}}{\sum_{i} w_{i}}
$$

The weight, $w_{i}$, is a decreasing function of the distance between the centroids of the element and its neighbors.

\subsection{Adjoint Sensitivity Analysis}

Adjoint sensitivity analysis is performed to calculate the gradient information required for the non-linear optimization process [34]. Let the functional of the objective function be defined as

$$
\Pi=\Theta\left(U_{f}(\rho), \rho\right)
$$

Where $\Theta$ be the response objective function, $\boldsymbol{U}_{\boldsymbol{f}}$ defines the displacement vector corresponding to the free degrees of freedom of the structure and $\rho$ is the vector of design variables. For the structure to be in equilibrium, the residual, $\boldsymbol{R}$, should be equal to zero for each deformed configuration.

$$
\boldsymbol{R}\left(\boldsymbol{U}_{\boldsymbol{f}}(\boldsymbol{\rho}), \boldsymbol{\rho}\right)=0
$$

The augmented Lagrangian function, $L$, is defined as

$$
L=\Theta+\lambda^{T} \boldsymbol{R}
$$

where $\lambda$ is a Lagrange multiplier. We can observe that $L$ is identically equal to the function of interest, $\Theta$, for all $\lambda$, since $R$ is zero, as shown in equation 12 . Hence, finding the sensitivity of $L$ with respect to the design variable, $\rho$, is the same as the sensitivity of the response function, $\Theta$. Using the chain rule of differentiation, we can write

$$
\frac{d L}{d \boldsymbol{\rho}}=\frac{\partial \Theta}{\partial \boldsymbol{\rho}}+\frac{\partial \Theta}{\partial \boldsymbol{U}_{\boldsymbol{f}}} \frac{d \boldsymbol{U}_{\boldsymbol{f}}}{d \boldsymbol{\rho}}+\lambda^{T}\left[\frac{\partial \boldsymbol{R}}{\partial \boldsymbol{U}_{\boldsymbol{f}}} \frac{d \boldsymbol{U}_{\boldsymbol{f}}}{d \boldsymbol{\rho}}+\frac{\partial \boldsymbol{R}}{\partial \boldsymbol{\rho}}\right]
$$


Here, the operator $\frac{\partial}{\partial \rho}$ captures the direct dependence of any function with respect to $\rho$. Whereas, the operator $\frac{d}{d \rho}$ captures the indirect dependence of a function with respect to variable $\rho$. Collecting all the implicit derivatives and rearranging equation 14

$$
\frac{d L}{d \boldsymbol{\rho}}=\frac{\partial \Theta}{\partial \boldsymbol{\rho}}+\lambda^{T} \frac{\partial \boldsymbol{R}}{\partial \boldsymbol{\rho}}+\left[\lambda^{T} \frac{\partial \boldsymbol{R}}{\partial \boldsymbol{U}_{\boldsymbol{f}}}+\frac{\partial \Theta}{\partial \boldsymbol{U}_{\boldsymbol{f}}}\right] \frac{d \boldsymbol{U}_{\boldsymbol{f}}}{d \boldsymbol{\rho}}
$$

Since $\lambda^{T}$ is arbitrary, we may select it to annihilate the coefficient of the the implicit term. The value of $\lambda^{T}$ that makes equation 15 independent of the implicit term can be written as:

$$
\lambda^{T}=-\left[\frac{\partial \boldsymbol{R}}{\partial \boldsymbol{U}_{\boldsymbol{f}}}\right]^{-T} \frac{\partial \Theta}{\partial \boldsymbol{U}_{\boldsymbol{f}}}=-\boldsymbol{K}_{\tan }^{-T}{\frac{\partial \Theta}{\partial \boldsymbol{U}_{\boldsymbol{f}}}}^{T}
$$

Here, $\boldsymbol{K}_{\text {tan }}$ is the tangent stiffness matrix and $\lambda$ is called the adjoint response. After determining the adjoint response, we can substitute its value in equation [15, to get the total derivative as:

$$
\frac{d L}{d \rho}=\frac{\partial \Theta}{\partial \rho}+\lambda^{T} \frac{\partial R}{\partial \rho}
$$

The structural optimization problem is solved using the Method of Moving Asymptotes (MMA) [35]. Using the initial starting values of the design variables and the gradients of the Lagrangian, the optimizer solves a convex approximation of the problem. The optimizer terminates when the first order Karush-Kuhn-Tucker (KKT) conditions are satisfied [36][37].

\section{Numerical Results}

\subsection{Bi-stable beam design}

The benchmark problem of bi-stable beam design has been analysed to understand the key aspects of topology optimization design for morphing structures. Additional details can be found in [27] and [25]. Figure1] shows the topology optimization design of a beam exhibiting snap-through behavior. The length and width of the beam are $6000 \mathrm{~mm}$ and $600 \mathrm{~mm}$, respectively. 


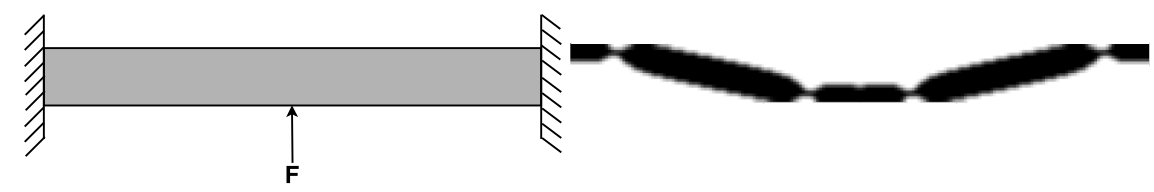

(a) Bi-stable beam design problem

(b) Optimized structure

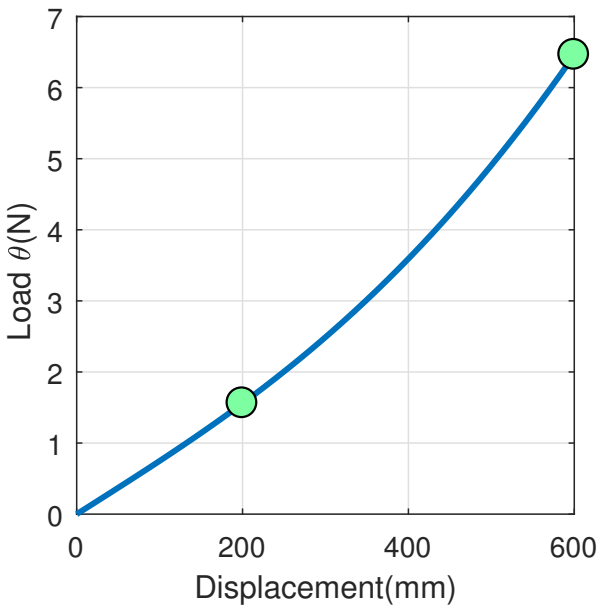

(c) Baseline beam

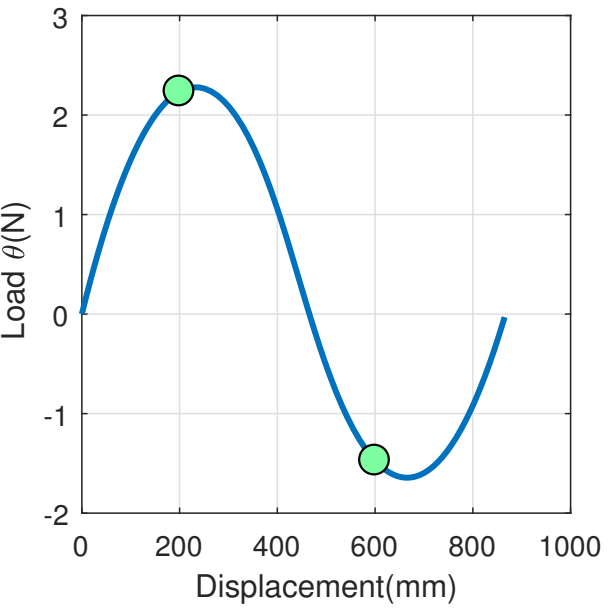

(d) Optimized bi-stable beam

Figure 1: The bi-stable beam design problem

The formal statement for optimization can be written as:

$$
\begin{array}{cl}
\underset{\boldsymbol{x}}{\operatorname{minimize}} & \theta_{2} \\
\text { subject to } & \theta_{1} \geq F_{\min }, \\
& M(\boldsymbol{\rho}) \leq M_{\max } .
\end{array}
$$

where $M$ represents the total mass of the structure. The objective is to minimize the externally applied force, $\theta_{2}$ at the prescribed displacement point $u_{2}$, subjected to a constraint on user defined force $\theta_{1}$, at some earlier displacement point, $u_{1}$, such that $\theta\left(u_{1}\right) \geq F_{\min }$. For this optimization, the value of $\theta_{1}$ is chosen to be $20 \%$ of the value of force required to displace the baseline structure by $u_{1}$. This is done to approximately control the level of displacement in the secondary stable position. We choose $\theta_{1}$ based on how we want the optimized force-displacement curve to look. Higher values of $\theta_{1}$ may sometimes fail to produce a bi-stable structure, whereas a very low value of $\theta_{1}$ may produce a structure with low stiffness. Generally a good range of $\theta_{1}$ values is 
$20 \%$ to $80 \%$ value of the force required to displace the baseline structure by $u_{1}$. The displacements $u_{1}$ and $u_{2}$ are chosen based on the desired deflection at the location of the input load. As shown in Figure 1d, the points $u_{1}$ and $u_{2}$ will be situated near the peak and valley (respectively) of the bi-modal force displacement curve. This fact can be used to approximately tailor the location of the structure's secondary equilibrium position. For the current design, $u_{1}$ and $u_{2}$ are chosen to be $200 \mathrm{~mm}$ and $600 \mathrm{~mm}$, respectively. The parameter $F_{\min }$ represents the minimum reaction force to ensure feasible structures with sufficient stiffness. It can be observed that for a structure to show bi-stability $\theta_{2}$ should be negative. The lower the value of $\theta_{2}$, the greater the force required to push the structure back to its baseline equilibrium state, and the greater will be the deflection of the airfoil will be. By constraining the force $\left(\theta_{1}\right)$ at the first deflection point, we ensure that the force-displacement response is bimodal to produce bistability.

\subsubsection{Mesh independence studies}

To validate the mesh independence of the solutions generated by the algorithm, we generate the results for the optimization problem described by equation (18) using different discretizations of the design domain with the same boundary conditions. We compare the solutions generated with the design domain discretized by an unstructured triangular mesh and structured quadrilateral meshes with different grid sizes. The boundary and loading conditions are the same as discussed in section 4.1. The baseline structure has a length of $6000 \mathrm{~mm}$ and a width of $600 \mathrm{~mm}$. The value of $u_{1}$ and $u_{2}$ chosen for the study are $100 \mathrm{~mm}$ and $400 \mathrm{~mm}$, respectively. This has been done to facilitate faster solution generation, since the main motivation of this study is to show mesh-independence of the algorithm. The radius of the density filter for all three discretizations has been kept constant at $120 \mathrm{~mm}$.

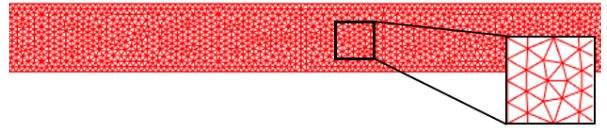

(a) Unstructured (CST) mesh

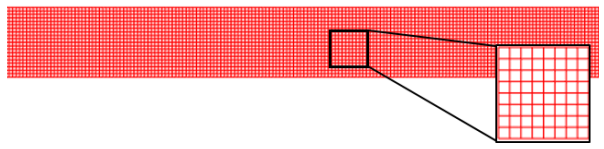

(c) Fine structured mesh

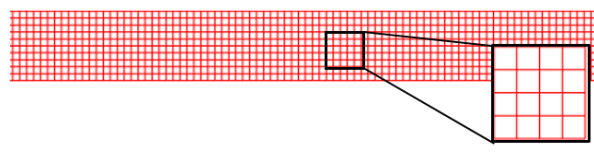

(e) Coarse structured mesh

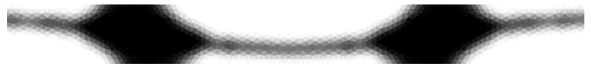

(b) Optimal design from mesh (a)

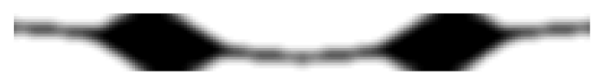

(d) Optimal design for mesh (c)

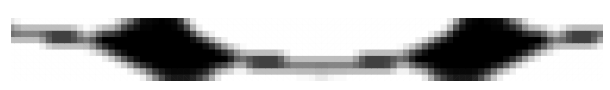

(f) Optimal design for mesh (e)

Figure 2: Mesh independence studies 
Figures $2 \mathrm{a} \& 2 \mathrm{~b}$ show the design domain discretized with an un-structured grid of 3028 constant-strain triangle (CST) elements and the corresponding optimized structure. Similarly, Figures 2c \& 2d show a design domain discretized with a structured grid of 2000 quadilateral elements and its corresponding optimized structure. Figures 2e \& 2f] show the design domain and the optimized structure for a structured grid of 500 quadrilateral elements. The above study confirms the mesh-independent behavior of the algorithm and the corresponding structures generated by the same. All the algorithms were implemented in a computer having the following specifications:

Table 1: Computer Hardware Specifications

\begin{tabular}{ll}
\hline \hline & \\
CPU make and model & Intel(R) Core(TM) i5-4590 CPU@3.30GHz \\
Maximum speed & $3.30 \mathrm{GHz}$ \\
Number of sockets & 1 \\
Number of cores & 4 \\
Number of logical processors & 4 \\
L1 cache & $256 \mathrm{~KB}$ \\
L2 cache & $1.0 \mathrm{MB}$ \\
L3 cache & $6.0 \mathrm{MB}$ \\
\hline \hline
\end{tabular}

The algorithms were written and executed using MATLAB, while the plotting of meshes and the design domains for the optimized solutions were done using MATLAB's parallel programming functionality with four cores.

\subsection{Bi-Stable Airfoil Design}

The key design aspects of a bi-stable beam are incorporated into the design of a bi-stable airfoil. The functional aspects of the wing spars have been simulated by fixing certain portions along the top and bottom surfaces. The boundary conditions and loading has been explained in Figure 3. The airfoil chosen for this study is based on NACA 0012 airfoil. The leading and trailing sections were removed to fit a structured grid. The airfoil is further modified to have a more favorable aspect ratio so that we can obtain a detailed design with fewer elements, and give the optimizer a larger domain in which to maneuver. The maximum thickness of the airfoil is changed by $178 \%$ to produce a modified NACA 0012 airfoil, suited to carry out the optimization analysis.

\subsubsection{Optimized airfoil with quadrilateral finite elements}

The first approach adopted involves the use of quadrilateral finite elements to mesh the airfoil design domain. The modified airfoil design domain is shown in Figure 3 . 


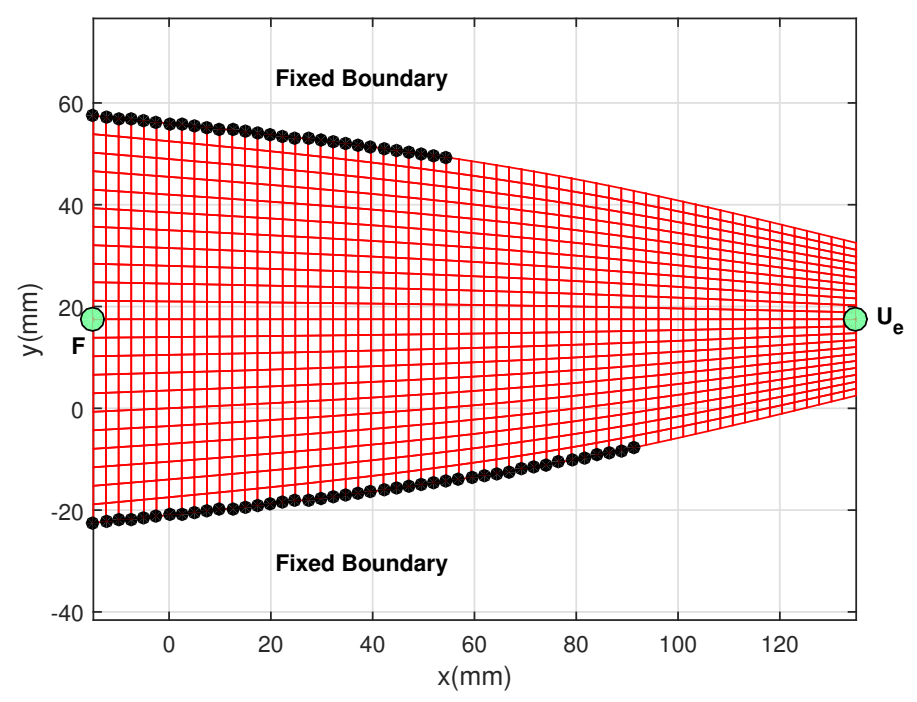

Figure 3: Design domain for the morphing airfoil optimization problem

The point of application of the input displacement and the point at which the deflection is desired are shown by the markers ' $\mathbf{F}$ ' and ' $U_{e}$ ' respectively. We want to minimize force $\left(\theta_{2}\right)$ required to deflect the trailing node $\left(U_{e}\right)$ by an amount greater than a minimum value $\left(U_{0}\right)$. To ensure proper structural rigidity at the bi-stable region, we constrain the force $\left(\theta_{1}\right)$ corresponding to the first displacement $\left(u_{1}\right)$ to be of value greater than, $F_{\min }$. The value of $F_{\min }$ is chosen to be $20 \%$ of the force corresponding to $u_{1}$ for the solid baseline design domain. The compliance constraint, $C(\boldsymbol{x})$ is used to ensure a continuous chain of material between the $U_{e}$ node and the ' $\mathbf{F}$ ' node. It also ensures the structure has sufficient stiffness required for its functionality. To compute the compliance constraint, $C(\boldsymbol{x})$, we fix the top and bottom surfaces, as represented by 'Fixed Boundary', and also the node $\mathrm{F}$, while displacing the trailing node, $U_{e}$, by 3 $\mathrm{mm}$. The volume constraint $V(\boldsymbol{x})$ controls what percent of the original design domain will form the optimized structure. Mathematically we can represent these as follows

$$
\begin{array}{cl}
\underset{\boldsymbol{x}}{\operatorname{minimize}} & \theta_{2} \\
\text { subject to } & \theta_{1} \geq F_{\min }, \\
& V(\boldsymbol{x}) \leq V_{0} \\
& C(\boldsymbol{x}) \geq C_{0} \\
& U_{e}(\boldsymbol{x}) \geq U_{0} .
\end{array}
$$

The design domain is discretized with 1342 quadrilateral finite elements, with 61 elements in the horizontal direction and 22 elements in the vertical direction. The computational performance data is provided in Table 2 Figure 4 shows the optimized material layout for this design problem. The compliance constraint was active in the 
final design and was satisfied for all the optimization results presented. Figure 5 a and $5 \mathrm{~b}$ show the optimized airfoil in its deformed configuration and the deformed finite element mesh respectively.

Table 2: Optimization Data for bistable airfoil design with quadrilateral elements \begin{tabular}{|l|l|l|l|}
\hline Convergence time(s) & Complexity (d.o.f) & Optimization Iterations & NR iterations \\
\hline
\end{tabular}

\begin{tabular}{|c|c|c|c|}
68886.4 & 2852 & 53 & 1082
\end{tabular}

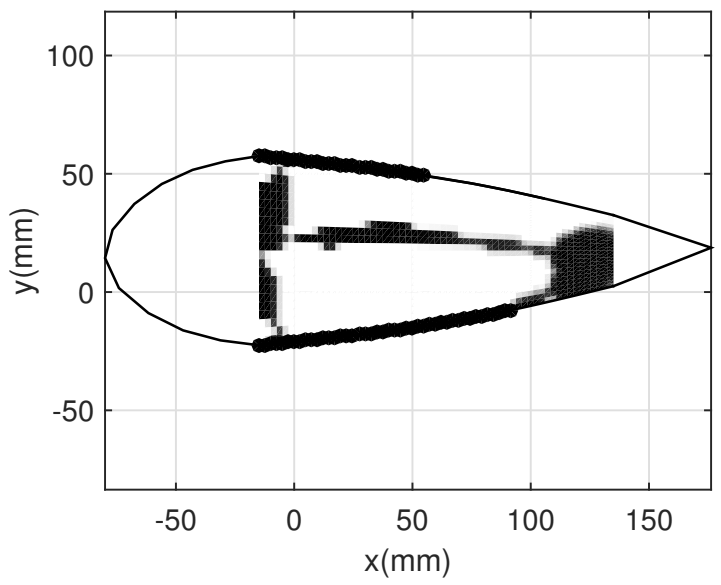

Figure 4: Optimized material layout 


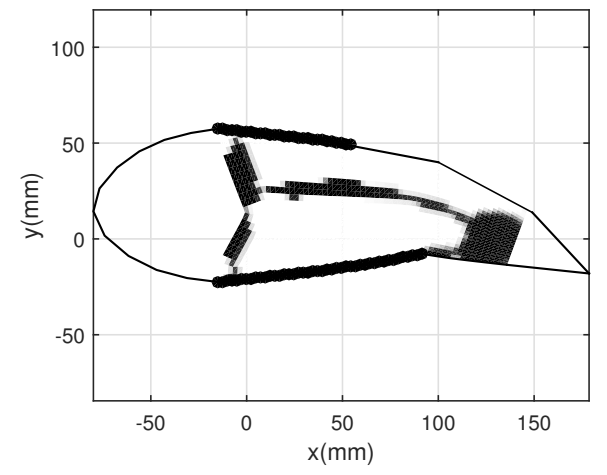

(a) Morphed structure

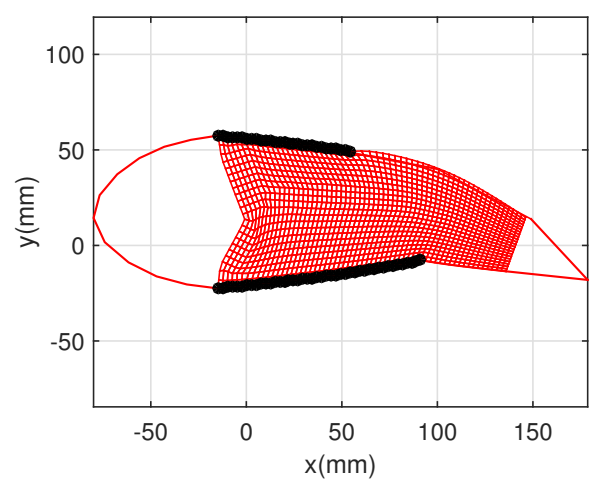

(b) Deformed mesh

Figure 5: Optimized airfoil with rectangular finite element mesh

Figure $5 \mathrm{~b}$ shows that the finite element mesh undergoes a considerable amount of distortion, which prevents us from using a minimum value of material density, $\rho_{\min }$, lower than 0.1, as it led to formation of degenerate elements. Another important point to consider is the stiffness contribution of the void elements. The elements present in the void regions contribute to the overall stiffness of the structure. This prevents us from accurately describing the force-displacement response of the structure. Therefore, to have a better understanding of actual structural response, we implement an algorithm to remove the void and near-void elements from the finite element mesh. The filter threshold value for the element removal process, $\rho_{\text {remove }}$, was chosen to be 0.1 . We then perform a non-linear analysis of the modified structure. Figure 6 shows the reduced mesh for the optimized design shown in Figure 4 in both the undeformed and deformed states. 


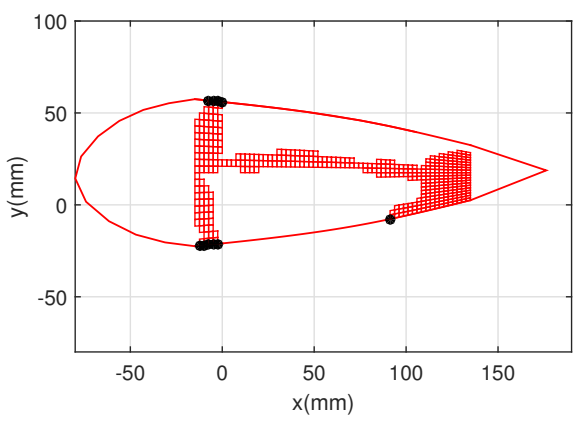

(a) Undeformed mesh

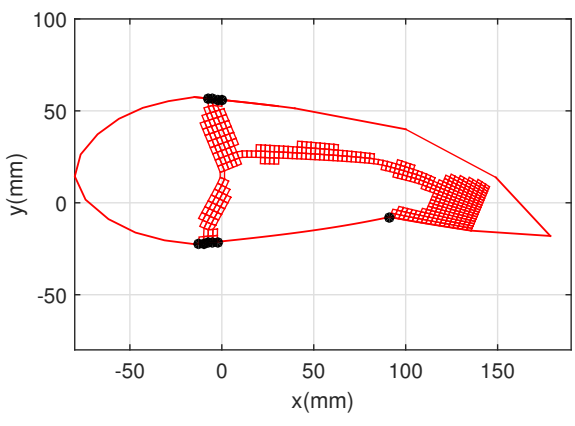

(b) Deformed mesh

Figure 6: Reduced mesh for bi-stable airfoil design

The maximum trailing edge deflection measured at node $U_{e}$ for the full finite element mesh was observed to be $12 \mathrm{~mm}$, with a maximum input deflection $u_{2}$ of $10 \mathrm{~mm}$. The trailing edge deflection increased to $13.6 \mathrm{~mm}$ for the same $u_{2}$ when reduced finite element mesh was used to carry out the structural analysis. Figure 7 shows the force-displacement response for the baseline structure, as well as for the optimized structure with both reduced and full finite element meshes. The force response of the baseline structure is monotonic, however, the optimizer redistributes material within the design domain to produce a bi-modal response representing snap-through instability. Figure $7 \mathrm{~b}$ shows that before removing the void elements the structure does not show bi-stability since the force always remains positive. However, after element removal the structural response changes considerably and shows bi-stability. 


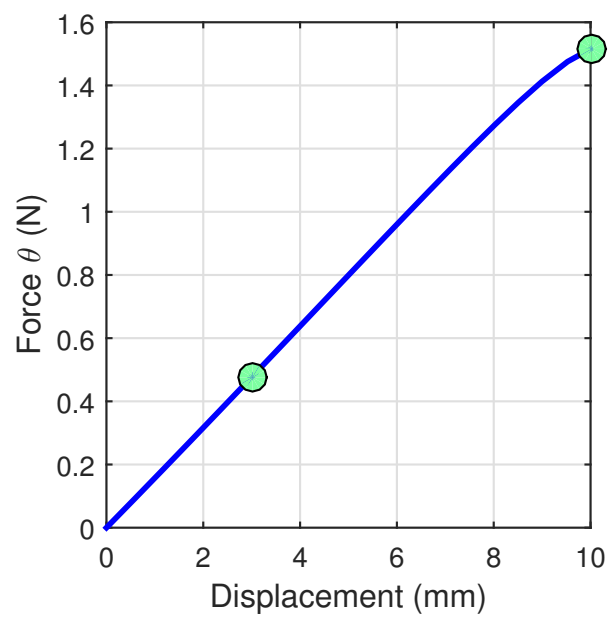

(a) Baseline structure

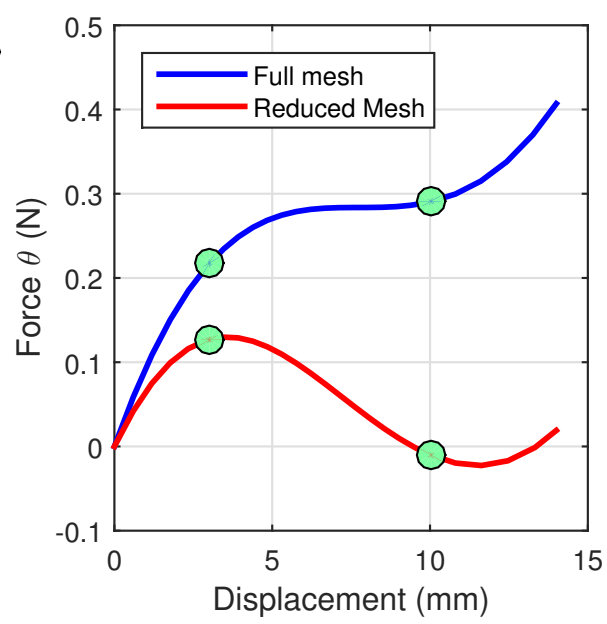

(b) Optimized structure

Figure 7: Force-displacement response of the bi-stable airfoil with rectangular finite elements

The optimization and structural parameters are summarized in the Table 3

Table 3: Optimization Parameters and Constraint Values for Quadrilateral Element Mesh

\begin{tabular}{ll}
\hline \hline & \\
Young's Modulus (MPa), $E_{0}$ & 15 \\
Minimum elemental density, $\rho_{\text {min }}$ & 0.1 \\
Volume Fraction, $V_{0}$ & 0.4 \\
Penalization parameter, $p$ & 3 \\
Maximum Input Displacement $(\mathrm{mm}), u_{2}$ & 10 \\
Minimum Tip-node Deflection $(\mathrm{mm}), \mathrm{U}_{e}$ & 13.6 \\
Displacement for Compliance Evaluation $(\mathrm{mm}), u_{c}$ & 3 \\
Complaince constraint $(\mathrm{N} . \mathrm{mm}), C_{0}$ & 0.7782 \\
Displacement Constraint $(\mathrm{mm}), U_{0}$ & 13 \\
Airfoil chord length (mm) & 300 \\
Airfoil maximum thickness $(\mathrm{mm})$ & 40 \\
\hline \hline
\end{tabular}

\subsubsection{Optimized Airfoil with Triangular Finite Elements}

In the previous section we used a structured quadrilateral finite element mesh to discretize the airfoil design domain. This required us to remove the trailing and leading edge of the airfoil. This modification restricted the optimizer from exploiting the full available design space and in turn this approach requires additional mechanisms to transfer deflections to the trailing edge of the airfoil. To overcome the above mentioned 
challenges, an unstructured triangular mesh is used to discretize the airfoil design domain.

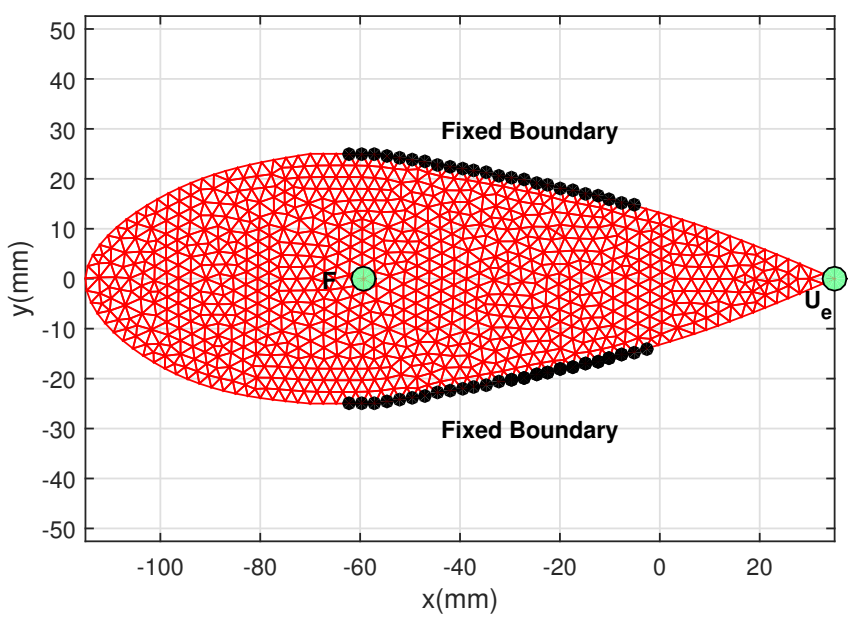

Figure 8: Boundary and loading conditions for bi-stable airfoil design domain

Figure 8 shows the nodes at which the input displacement is applied, the boundary conditions along with the node at which the deflection is desired for the morphing application. The objective function and the constraints for the optimization are the same as used for the quadrilateral elements. We constrain the trailing edge node $\left(U_{e}\right)$ to deflect by an amount greater than a minimum value, $U_{o}$ and minimize the actuating force $\theta_{2}$. To ensure a continuous chain of material between the trailing edge and the point of application of the actuating force, a compliance constraint is enforced. It also ensures that the structure is stiff enough to fulfill its functional requirements. The fixed boundary conditions are imposed on the upper and lower airfoil surfaces to imitate the behavior of supports and spars. Mathematically, we can represent these as follows

$$
\begin{array}{cl}
\underset{\boldsymbol{x}}{\operatorname{minimize}} & \theta_{2} \\
\text { subject to } & \theta_{1} \geq F_{\min }, \\
& V(\boldsymbol{x}) \leq V_{0} \\
& C(\boldsymbol{x}) \geq C_{0} \\
& U_{e}(\boldsymbol{x}) \geq U_{0} .
\end{array}
$$

where $F_{\min }$ is chosen to be $20 \%$ of the force evaluated at the first displacement position $\left(u_{1}\right)$ for the baseline structure. The design domain is discretized with 1600 triangular elements. To avoid local minima, we employ a continuation method [38] in which the SIMP penalization parameter, $p$, is increased in steps, as a function of iteration number, $j$, such that.

$$
p(j)=\max (3.0, \min (\operatorname{ceil}[j / 5], 5.0)), j=1,2, \ldots
$$


The minimum density value $\left(x_{\min }\right)$ for the entire design domain changes whenever the penalization parameter increases. To ensure a continuous mapping from the old minimum density to new minimum density value, whenever there is a change in penalization parameter, $p$, a mapping function is formulated.

$$
\boldsymbol{x}_{\min p^{n+1}}=A \boldsymbol{x}_{\min p^{n}}+B
$$

Here, A and B are constant mapping parameters that have been used to shift the minimum element density from the previous values, when the penalization parameter was, $p^{n}$, to the new values when the penalization parameter becomes $p^{n+1}$. These values are given by,

$$
\begin{aligned}
& A=\frac{1-\sqrt[n+1]{E_{\text {min }_{e}}}}{1-\sqrt[n]{E_{\min _{e}}}} \\
& B=1-A
\end{aligned}
$$

where $E_{\text {mine }}=1 \times 10^{-3}$ for all values of $\rho_{\min }$. Figure 9 shows the optimized airfoil. Table 4 lists the computational performance data for the design problem. Figure 10a and $10 \mathrm{~b}$ shows the deformed optimized airfoil and the deformed finite element mesh, respectively. We can observe the high amount of distortion undergone by the finite element mesh.

\section{Table 4: Optimization Data}

\begin{tabular}{|c|c|c|c|}
\hline Convergence time(s) & Complexity (d.o.f) & Optimization Iterations & NR iterations \\
\hline 7877.9 & 1846 & 126 & 2956 \\
\hline
\end{tabular}

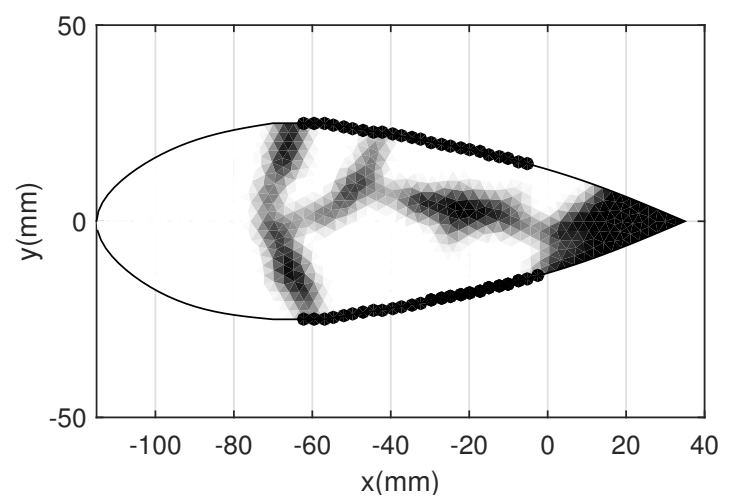

Figure 9: Optimized material layout

As previously mentioned, to obtain an accurate description of the force-displacement response of the optimized structure, we remove the void and 
near-void elements. We use a filter threshold value of 0.45 for the element removal process. Figure 11 shows the reduced finite element meshes for the undeformed and deformed configurations of the airfoil. Figure 12 shows the force-displacement response of the optimized airfoil with both full and reduced finite element meshes. We observe that the structure does not show bi-stability with the full mesh. When then void elements are removed we get a bi-stable structure, but for a displacement less than the maximum input displacement of $15 \mathrm{~mm}$. The structure also exhibits characteristics of snap-back instability.

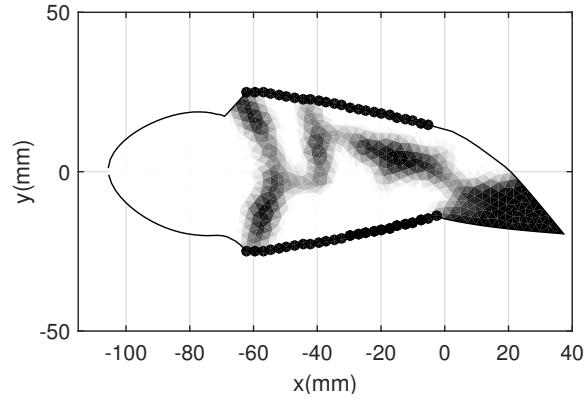

(a) Optimized airfoil

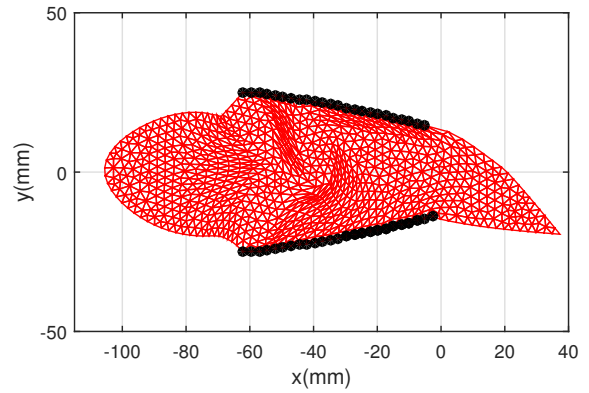

(b) Deformed mesh

Figure 10: Optimized airfoil and deformed mesh

For a maximum input deflection, $u_{2}$, of $15 \mathrm{~mm}$, structural analysis carried out with the full finite element mesh produces a trailing edge deflection, $U_{e}$, of $16 \mathrm{~mm}$. While, reduced finite element mesh analysis, for the same input deflection, produces a trailing edge deflection of $21 \mathrm{~mm}$. The black markers in Figure 11 show the reduced boundary conditions.

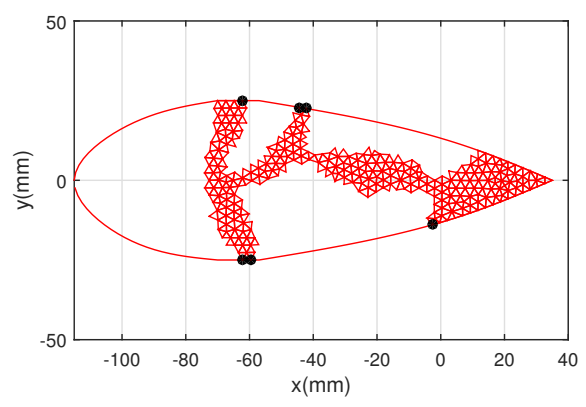

(a) Undeformed mesh

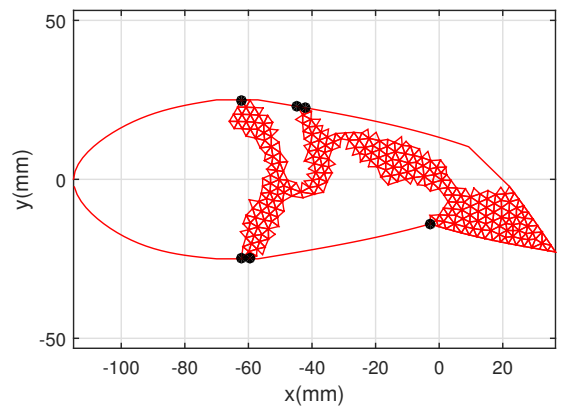

(b) Deformed mesh

Figure 11: Reduced mesh for bi-stable airfoil design 


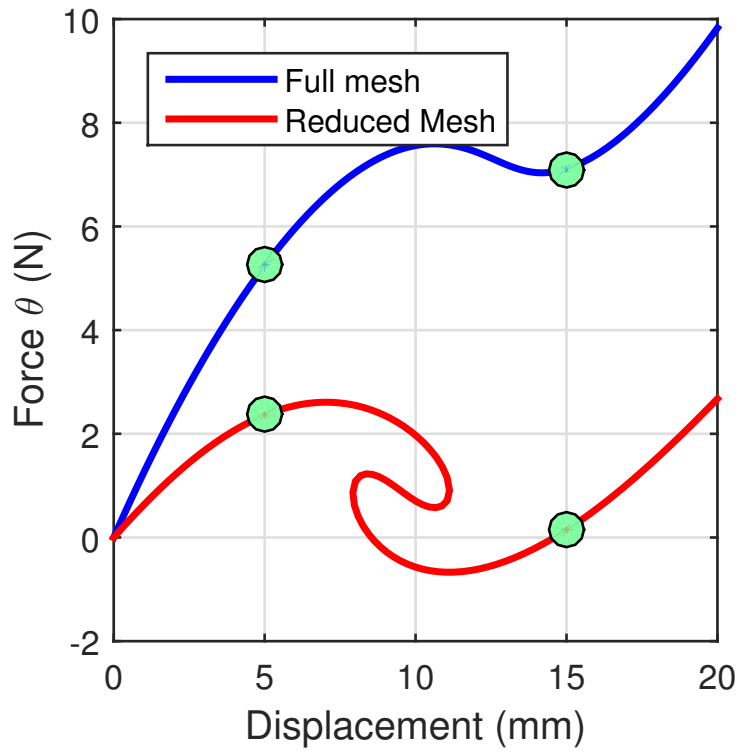

Figure 12: Force-displacement plot for optimized airfoil with reduced and full finite element meshes

Table 5: Optimization and design Parameters

\begin{tabular}{llll}
\hline \hline & & & \\
Young's Modulus (MPa), $E_{0}$ & 15 & & \\
Minimum elemental density, $\rho_{\text {min }}$ & 0.1 & 0.177 & 0.251 \\
Penalization Parameter, $p$ & 3 & 4 & 5 \\
Volume Fraction, $V_{0}$ & 0.4 & & \\
Maximum Input Displacement (mm), $u_{2}$ & 15 & \\
Minimum trailing edge Deflection(mm), $\mathrm{u}_{0}$ & 21 & \\
Complaince Constraint (N mm), $C_{0}$ & 12.33 \\
Displacement for Compliance Evaluation $(\mathrm{mm}), u_{c}$ & 10 \\
Displacement Constraint (mm), $U_{0}$ & 16 \\
Airfoil Chord Length (mm) & 150 & \\
Airfoil maximum thickness (mm) & 50 & \\
\hline \hline
\end{tabular}

\subsubsection{Alternative parameter design}

To make the algorithm stay away from potential snap-back issues, as observed in the force-displacement response curve shown in Figure 12, the optimization parameter, $F_{\min }$ (which governs the stiffness value of the bistable region) was reduced to half of its original value. The other design and optimization parameters were kept the same. Figure 13a shows the optimized structure corresponding to the alternative optimization 
problem. Table 6represents the computational performance data for the same problem. Figure $13 \mathrm{~b}$ shows the deformed optimized airfoil configuration.

\section{Table 6: Optimization Data}

\begin{tabular}{|c|c|c|c|}
\hline Convergence time(s) & Complexity (d.o.f) & Optimization Iterations & NR iterations \\
\hline 16471 & 1846 & 274 & 6389 \\
\hline
\end{tabular}

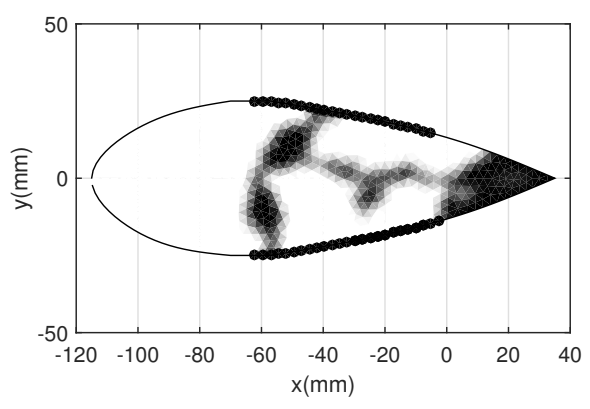

(a) Optimized material distribution

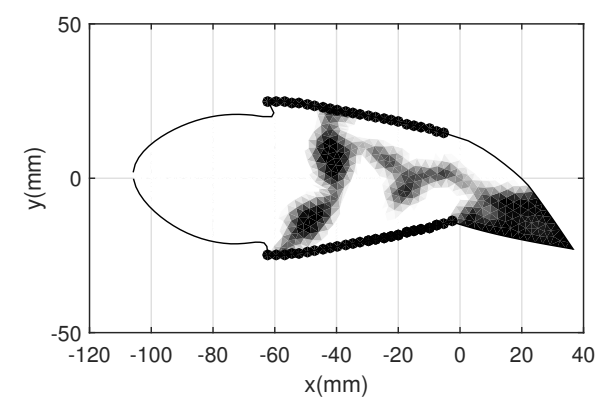

(b) Morphed configuration

Figure 13: The Alternative optimization problem

Figure 14 shows the finite element mesh distortion. Figure 15 shows the reduced finite element mesh for the optimized airfoil in undeformed and deformed configurations respectively. For a maximum input deflection, $u_{2}$, of $15 \mathrm{~mm}$, the trailing edge, $U_{e}$, deflects by $19 \mathrm{~mm}$ for a full finite element mesh. However, with the reduced finite element mesh, the maximum trailing edge deflection, with the same $u_{2}$, is observed to be $25.8 \mathrm{~mm}$. Thus, the morphing mechanism displays a geometric advantage of 1.98 , much higher than the previous design. 


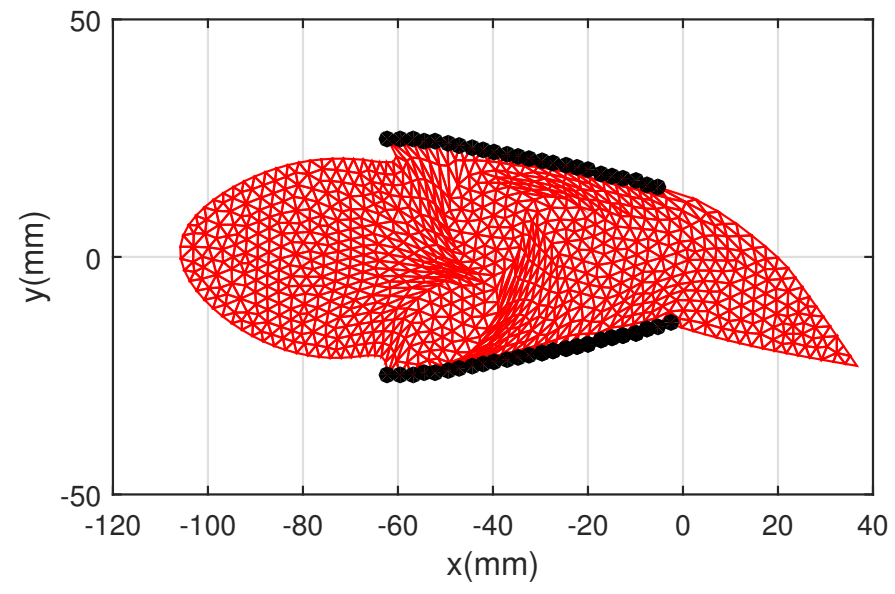

Figure 14: Finite element mesh distortion

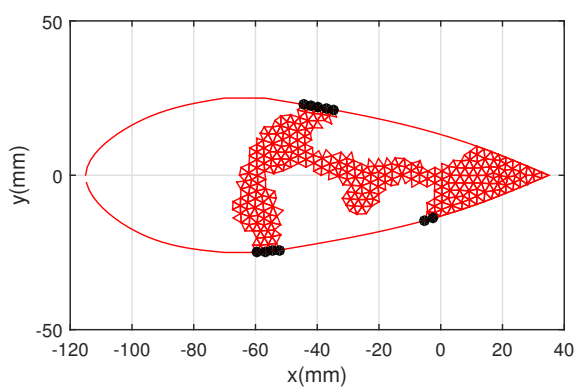

(a) Undeformed mesh

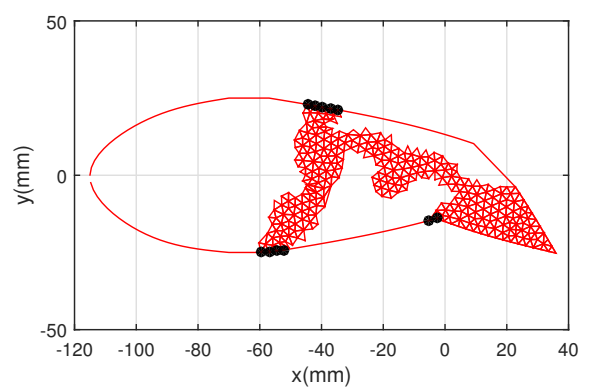

(b) Deformed mesh

Figure 15: Reduced meshes

Figure 16 shows the force-displacement curves corresponding to the baseline structure and optimized airfoil structure. The force-displacement response of the baseline structure is monotonic. The optimizer tailors the response of the structure to produce a bi-modal curve exhibiting snap through characteristics. After removing the void elements from the finite element mesh, we obtain a bi-stable morphing airfoil structure. The plot produced with the reduced finite element mesh confirms that the optimized design obtained is actually bi-stable because the curve dips below the $\mathrm{x}$-axis when the deflection exceeds the second displacement position $\left(u_{2}\right)$ value. 


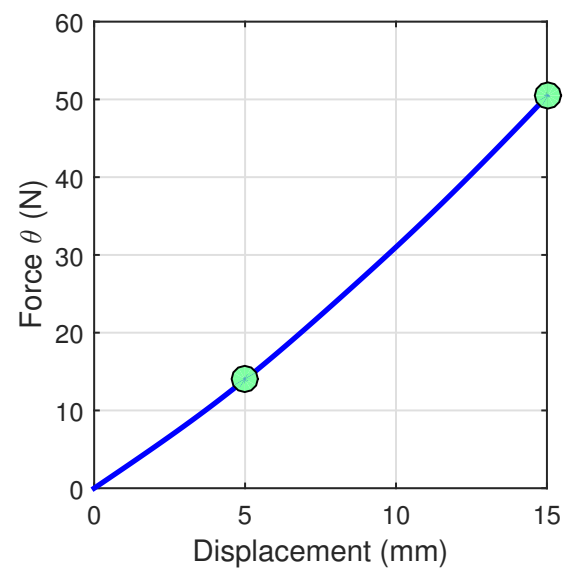

(a) Baseline structure

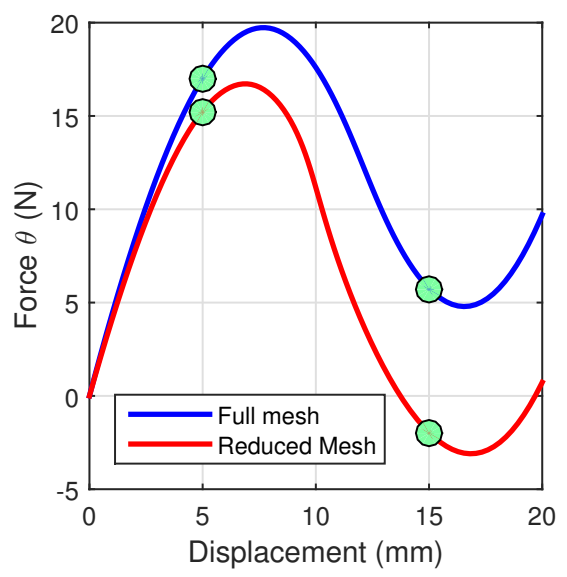

(b) Optimized structure

Figure 16: Force-displacement response of the bi-stable airfoil with triangular finite elements for the alternative optimization problem

The element-density removal threshold for Figure 15] is 0.2. The effect of different removal thresholds on the bi-stable behaviour of the structures has been shown in Figure 17 .

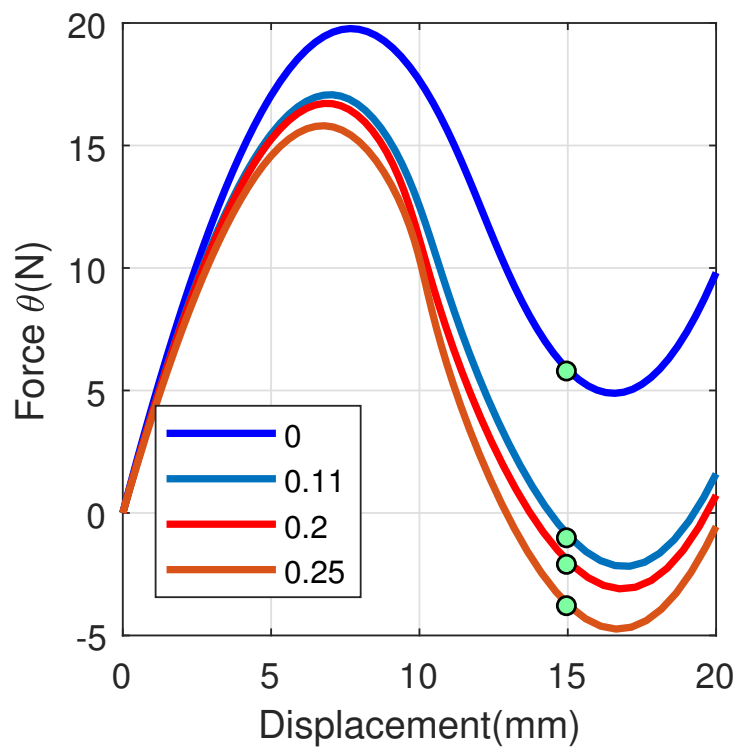

Figure 17: Force-displacement plots with different element removal thresholds 


\section{Additional Examples}

To explore additional benchmark problems, the optimization framework, as described in the previous sections, was applied to design a bi-stable gripper and a bi-stable inverter. The problem formulation is similar to equation 20].

\subsection{Bi-stable gripper}

Figure 18 shows the design domain along with the boundary conditions for the bi-stable gripper design problem. The design domain, of size $100 \mathrm{~mm} \times 100 \mathrm{~mm}$, is meshed with a structured grid of 3360 quadrilateral elements with 60 elements in each directions. The size of the cut-out in the $x$ and $y$ directions is $25 \%$ of the length of the design domain in the corresponding directions. The input displacements $u_{1}$ and $u_{2}$ are $2.5 \mathrm{~mm}$ and $6 \mathrm{~mm}$ respectively. The constraint $U_{0}$ as in equation 20 is $1 \mathrm{~mm}$.

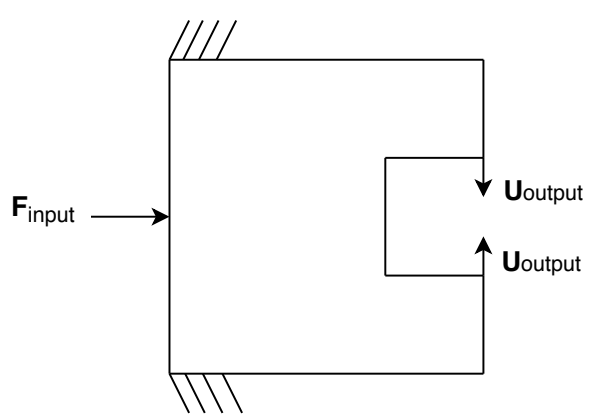

Figure 18: Bi-stable gripper design domain and boundary conditions 


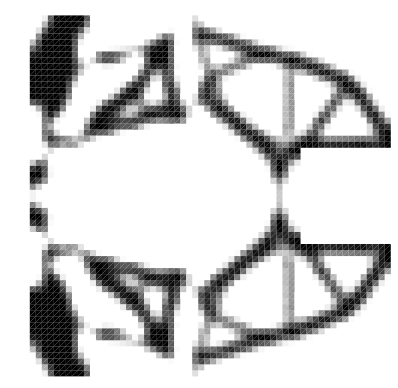

(a) Optimized structure

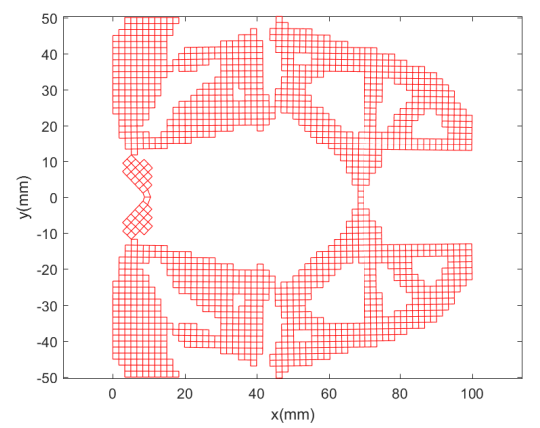

(c) Deformed mesh

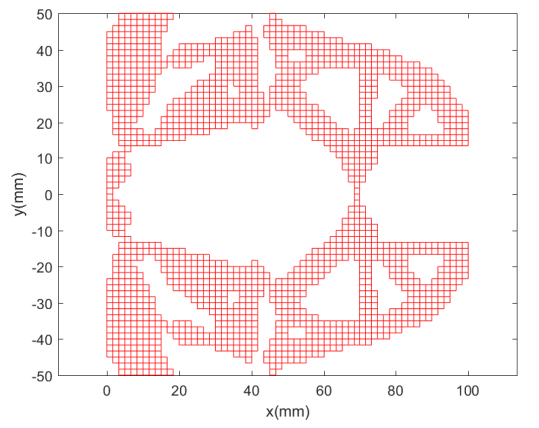

(b) Reduced mesh

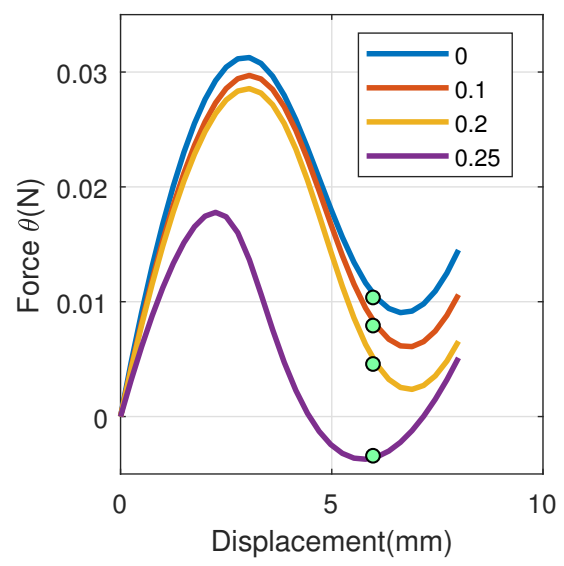

(d) Force-displacement plots

Figure 19: Bi-stable gripper design solutions

Figure 19 shows the optimized structure obtained from the algorithm. Figures $19 \mathrm{a}$ $19 \mathrm{~b} \& 19 \mathrm{c}$ represent the optimized material distribution layout and the corresponding undeformed and deformed reduced meshes respectively. Figure 19d shows the force-displacement plots for different values of element-removal density thresholds. We can infer that for a removal threshold value of 0.25 the structure is indeed bi-stable.

\subsection{Bi-stable inverter}

Figure 20 shows the design domain and the boundary conditions for bi-stable inverter design problem. The baseline structure has dimensions of $100 \mathrm{~mm} \times 100$ $\mathrm{mm}$ and is discretized using 3600 elements with 60 elements in each directions. The input displacements required for a bi-stable design are $u_{1}=2 \mathrm{~mm}$ and $u_{2}=5 \mathrm{~mm}$. The constraint displacement $U_{0}$ is $1 \mathrm{~mm}$. 


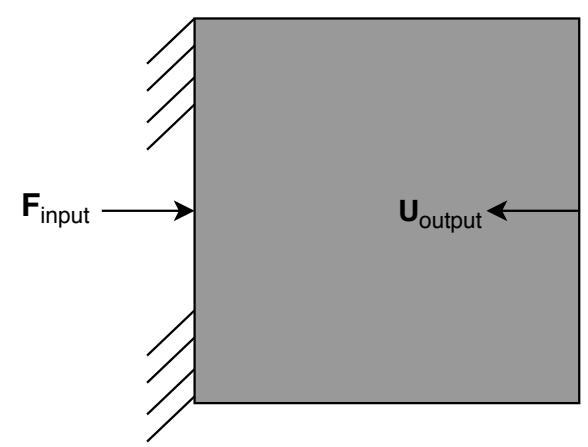

Figure 20: Bi-stable inverter design domain and boundary conditions

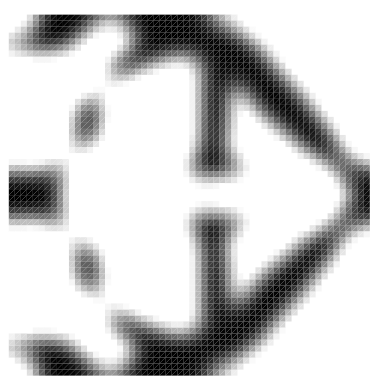

(a) Optimized structure

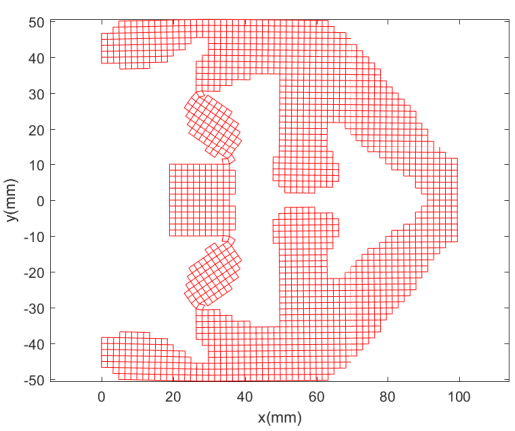

(c) Deformed mesh

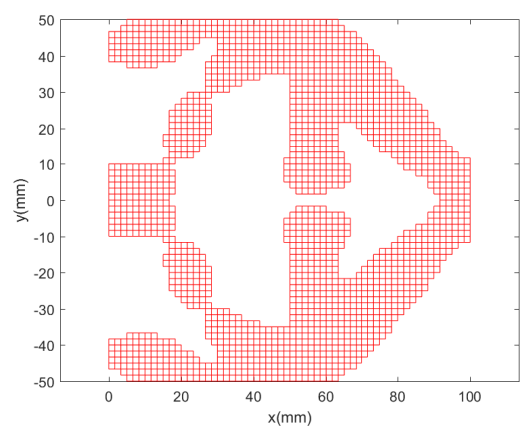

(b) Reduced mesh

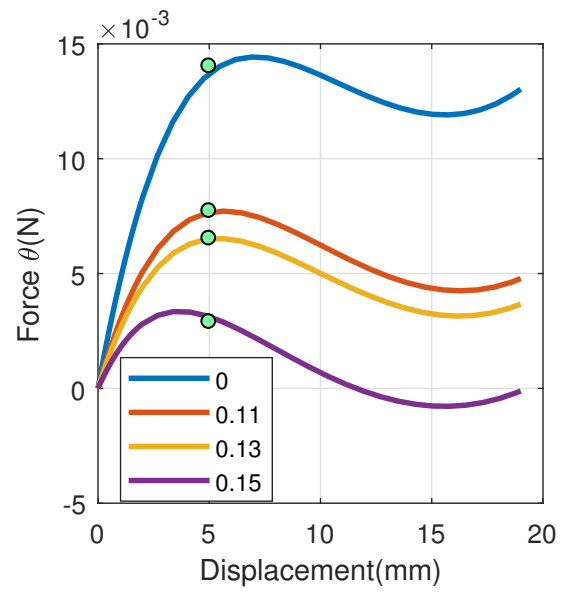

(d) Force-displacement plots

Figure 21: Bi-stable inverter design solutions

Figures 21a 21b \& 21c show the optimized material distribution layout along 
with the undeformed and deformed reduced meshes for the bi-stable inverter design problem. Figure 21d shows the force-displacement plots for different values of element-removal density thresholds. It can be seen that for a removal threshold value of 0.15 the structure is bi-stable.

\section{Discussion}

The present study gave an insight into the relative stability of the triangular and rectangular finite elements. It was observed that the maximum displacement at the input node, $u_{2}$, for the design domain meshed with structured quadrilateral finite elements was restricted to $10 \mathrm{~mm}$, since a higher displacement value made the tangent stiffness matrix ( $\boldsymbol{K}_{\tan }$ in equation 6) singular, due to high mesh distortion. While, for the design domain meshed with an unstructured triangular finite elements, the maximum displacement, $u_{2}$, was $15 \mathrm{~mm}$, with all the other optimization and design parameters kept same. This, along with the complex airfoil design domain, supported the use of an unstructured grid to obtain accurate optimization and structural analysis results. The filter radius can have a significant effect on the instability introduced due to the snap-back effect while performing structural analysis during the optimization process. We found that for fixed optimization and design parameters, increasing the filter radius, $R$, eliminates the snap-back instability.

\subsection{Design Validation}

To validate the results obtained from the topology optimization process, the proposed design solutions were prototyped using Objet260 Connex 3D printer and a performance comparision was made between the design solutions obtained computationally and the 3D printed designs. The topology optimization results were converted into CAD models using SolidWorks. The CAD model corresponding to the topology optimization result obtained for the structured quadrilateral mesh is shown in Figure 22 and the corresponding 3-D printed structures are shown in Figure 23.

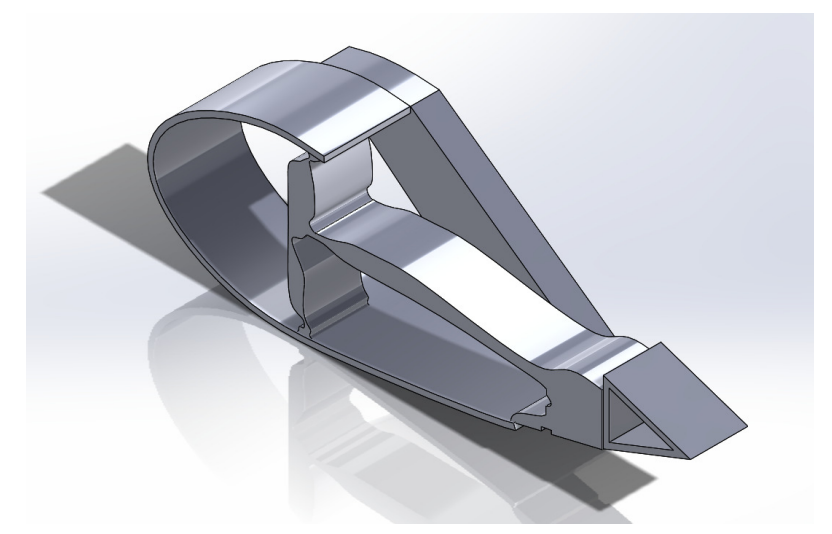

Figure 22: CAD model corresponding to quadrilateral mesh optimization result 


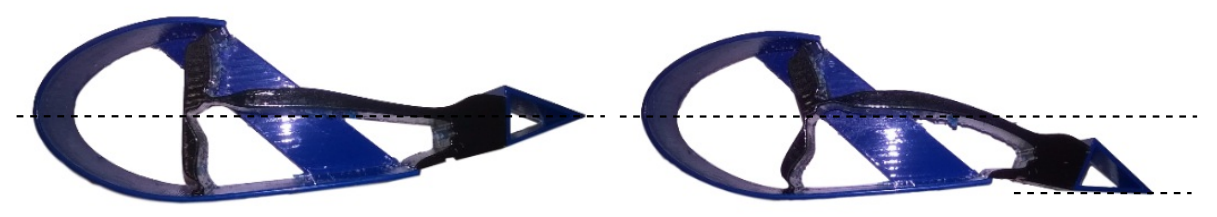

(a) Unmorphed configuration

(b) Morphed target configuration

Figure 23: 3-D printed optimized structures

The trailing edge deflection obtained with the finite-element analysis of the optimized structure with reduced mesh was $13.6 \mathrm{~mm}$. The 3D printed structure shows a trailing edge deflection of $10 \mathrm{~mm}$. The CAD model for the 'alternative optimization' problem is shown in Figure 24. The corresponding 3-D printed structures are shown in Figure 25.

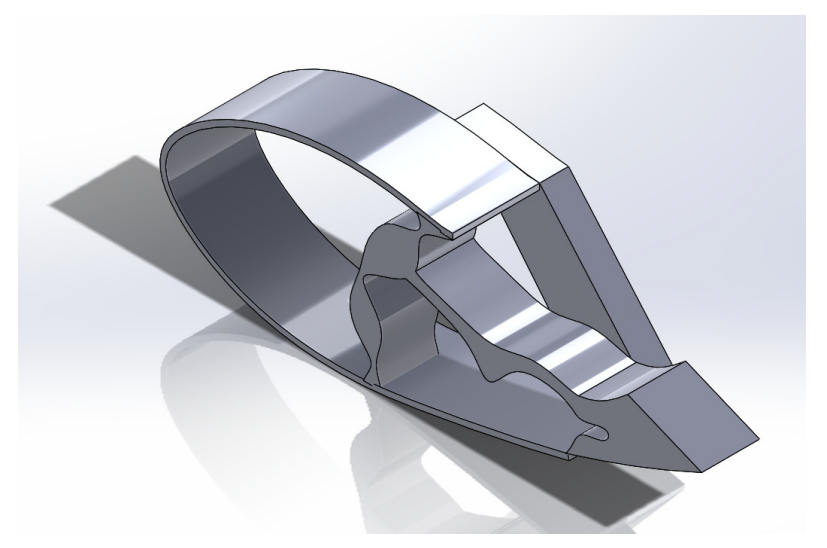

Figure 24: CAD model corresponding to triangular mesh optimization result 


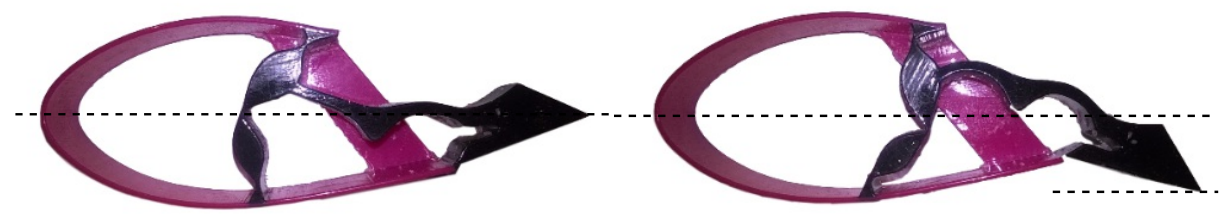

(a) Unmorphed configuration

(b) Morphed target configuration

Figure 25: 3-D printed optimized structures

The maximum trailing edge deflection for the numerically produced optimized structure was $25.8 \mathrm{~mm}$. The 3D printed structure showed a maximum trailing edge deflection of $22 \mathrm{~mm}$.

\section{Conclusion}

The present study successfully proposed a novel camber morphing mechanism, capable of producing a bi-stable airfoil, using topology optimization. The design uses the concept of bi-stability as a source of actuation for the morphing mechanism and for maintaining the deformed configuration of the structure. Previous works on topology optimization for bi-stability were restricted chiefly to the design of structures derived directly from the classic beam design problem. The contribution of this study and the results obtained are significant since they attempt to provide an alternative approach to design mechanisms by linking the bistable response of a structure to an output displacement at a separate location from the input node thereby, treating the design as a mechanism. The input and output nodes does not necessarily have to be on the edges of the design domain but can be placed anywhere. The optimal layout of material throughout the design domain was formulated as a continuous material distribution problem following the SIMP approach. The structure was optimized for bi-stability subject to resource constraints and a minimum trailing edge deflection. The structural response due to the actuating force was computed using a hyperelastic finite element formulation that accounts for large displacements. The design sensitivities were computed analytically using the adjoint method. Two different types of design domains with different boundary and optimization parameters were presented. In one approach, a structured quadrilateral grid was used to mesh a part of the airfoil domain. In the other approach, the airfoil was meshed using an unstructured triangular grid. Both the design approaches were analyzed and compared. Through optimization, we were able to tailor the monotonic response of a structure to exhibit snap-through behavior. After removing void elements, which prevented us from modeling the accurate structural response, a bi-stable structure was produced. The results showed that topology optimization can be used to exploit the geometric nonlinearity of structures to design morphing mechanisms. The proposed design algorithm was implemented on problems involving 
both structured (quadrilateral) and unstructured (triangular) meshes, and the algorithm was shown to successfully produce converged bi-stable designs in both cases. The non-linear material modeling along with the numerous constraints imposed makes the algorithm susceptible to divergence issues necessitating a parametric study of input variables before obtaining optimal solutions. The design process also requires specific user inputs regarding the boundary conditions. Future work will attempt to optimize the boundary conditions along with the material distribution to make the algorithm determine the optimal design with minimal human input. The structures, though predictive in nature to certain extent, are optimal. The direct wind-tunnel testing of the computationally obtained results are not possible because of the need for other components like a flexible skin over the airfoil surface and other actuating parts. Future work will also include aeroelastic coupling to observe the effects of aerodynamic loading on the structure.

\section{Acknowledgment}

The authors wish to acknowledge the financial support of the National Science Foundation under grant \# 1663566.

\section{References}

[1] Barbarino, S., Bilgen, O., Ajaj, R. M., Friswell, M. I., and Inman, D. J., “A review of morphing aircraft," Journal of intelligent material systems and structures, Vol. 22, No. 9, 2011, pp. 823-877. http://doi.org/10.1177/1045389X11414084

[2] Maute, K. K., and Reich, G. W., "Integrated multidisciplinary topology optimization approach to adaptive wing design," Journal of Aircraft, Vol. 43, No. 1, 2006, pp. 253-263.

http://doi.org/10.2514/1.12802

[3] Reich, G., and Sanders, B., "Introduction to morphing aircraft research," Journal of Aircraft, Vol. 44, No. 4, 2007, pp. 1059-1059.

http://dx.doi.org/10.2514/1.28287

[4] Thill, C., Etches, J., Bond, I., Potter, K., and Weaver, P., "Morphing skins," The Aeronautical Journal, Vol. 112, No. 1129, 2008, pp. 117-139. http://doi.org/10.1017/S0001924000002062

[5] Roth, B., Peters, C., and Crossley, W., "Aircraft sizing with morphing as an independent variable: motivation, strategies and investigations," Aircraft Technology, Integration, and Operations (ATIO), AIAA, Oct. 2002.

[6] Friswell, M. I., and Inman, D. J., "Morphing concepts for UAVs," Proceedings of the 21st international unmanned air vehicle systems conference., April, 2006. 
[7] Woods, B. K., Bilgen, O., and Friswell, M. I., "Wind tunnel testing of the fish bone active camber morphing concept," Journal of Intelligent Material Systems and Structures, Vol. 25, No. 7, 2014, pp. 772-785. http://dx.doi.org/10.1177/1045389X14521700

[8] Woods, B. K. S., and Friswell, M. I., “ Preliminary investigation of a fishbone active camber concept," ASME 2012 Conference on Smart Materials, Adaptive Structures and Intelligent Systems, American Society of Mechanical Engineers, Vol. 2, September, 2012, pp. 555-563. http://doi.org/10.1115/SMASIS2012-8058

[9] Diaconu, C. G., Weaver, P. M., and Mattioni, F., "Concepts for morphing airfoil sections using bi-stable laminated composite structures," Thin-Walled Structures, Vol. 46, No. 6, 2008, pp. 689-701. http://dx.doi.org/10.1016/j.tws.2007.11.002

[10] Pontecorvo, M. E., Barbarino, S., Murray, G. J., and Gandhi, F. S., "Bistable arches for morphing applications," Journal of Intelligent Material Systems and Structures, Vol. 24, No. 3, 2013, pp. 274-286. http://doi.org/10.1177/1045389X12457252

[11] Saggere, L., and Kota, S., "Static shape control of smart structures using compliant mechanisms," AIAA journal, Vol. 37, No. 5, 1999, pp. 572-578. http://dx.doi.org/10.2514/2.775

[12] Bartley-Cho, J. D., Wang, D. P., Martin, C. A., Kudva, J. N., and West, M. N., "Development of high-rate, adaptive trailing edge control surface for the smart wing phase 2 wind tunnel model," Journal of Intelligent Material Systems and Structures, Vol. 15, No. 4, 2004, pp. 279-291. http://dx.doi.org/10.1177/1045389X04042798

[13] Sofla, A. Y. N., Elzey, D. M.,and Wadley, H. N. G., "Cyclic degradation of antagonistic shape memory actuated structures," Smart Materials and Structures, Vol. 17, No. 2, 2008, pp. 25014. http://dx.doi.org/10.1088/0964-1726/17/2/025014

[14] Sofla, A. Y. N., Elzey, D. M., and Wadley, H. N. G., "Two-way antagonistic shape actuation based on the one-way shape memory effect," Journal of Intelligent Material Systems and Structures, Vol. 19, No. 9, 2007, pp. 1017-1027. http://doi.org/10.1177/1045389X07083026

[15] Sofla, A. Y., Elzey, D. M., and Wadley, H. N., "An antagonistic flexural unit cell for design of shape morphing structures," ASME 2004 International Mechanical Engineering Congress and Exposition, American Society of Mechanical Engineers, January, 2004, pp. 261-269. http://dx.doi.org/10.1115/IMECE2004-62149

[16] Yang, D., Mosadegh, B., Ainla, A., Lee, B., Khashai, F., Suo, Z., Bertoldi, K. and Whitesides, G.M., " Buckling of elastomeric beams enables actuation of soft 
machines," Advanced Materials, Vol. 27, No. 41, 2015, pp. 6323-6327.

http://dx.doi.org/10.1002/adma.201503188

[17] Rudykh, S. and Bhattacharya, K., "Snap-through actuation of thick-wall electroactive balloons," International Journal of Non-Linear Mechanics, Vol. 47, No. 2, 2012, pp. 206-209.

http://dx.doi.org/10.1016/j.ijnonlinmec.2011.05.006

[18] Overvelde, J.T., Kloek, T., D'haen, J.J. and Bertoldi, K., "Amplifying the response of soft actuators by harnessing snap-through instabilities," Proceedings of the National Academy of Sciences, Vol. 112, No. 35, 2015, pp. 10863-10868.

http://dx.doi.org/10.1073/pnas.1504947112

[19] Bruns, T.E. and Tortorelli, D.A., "Topology optimization of non-linear elastic structures and compliant mechanisms," Computer Methods in Applied Mechanics and Engineering, Vol. 190, No. 26, 2001, pp. 3443-3459.

http://doi.org/10.1016/S0045-7825(00)00278-4

[20] Nishiwaki, S., Frecker, M.I., Min, S. and Kikuchi, N., "Topology optimization of compliant mechanisms using the homogenization method," International Journal for Numerical Methods in Engineering, Vol. 42, No. 3, 1998, pp. 535-559. http://dx.doi.org/10.1002/(SICI) 1097-0207(19980615)42:3<535: : AID-NME372>3.0.CO;2-J

[21] Frecker, M., Kikuchi, N. and Kota, S., "Topology optimization of compliant mechanisms with multiple outputs," Structural Optimization, Vol. 17, No. 4, 1999, pp. 269-278.

http://dx.doi.org/10.1007/BF01207003

[22] Larsen, U.D., Sigmund, O. and Bouwstra, S., "Design and fabrication of compliant micromechanisms and structures with negative Poisson's ratio," Micro Electro Mechanical Systems, 1996, MEMS'96, Proceedings. An Investigation of Micro Structures, Sensors, Actuators, Machines and Systems, IEEE, Feb., 1996, pp. 365-371.

http://doi.org/10.1109/MEMSYS.1996.494009

[23] Sigmund, O., "On the design of compliant mechanisms using topology optimization," Journal of Structural Mechanics, Vol. 25, No. 4, 1997, pp. 493-524. http://dx.doi.org/10.1080/08905459708945415

[24] Sigmund, O., "A 99 line topology optimization code written in Matlab," Structural and multidisciplinary optimization, Vol. 21, No. 2, 2001, pp. 120-127. http://dx.doi.org/10.1007/s001580050176

[25] Bruns, T.E., Sigmund, O. and Tortorelli, D.A., "Numerical methods for the topology optimization of structures that exhibit snap-through," International Journal for Numerical Methods in Engineering, Vol. 55, No. 10, 2002, pp. 1215-1237.

http://doi.org/10.1002/nme.544 
[26] Ramos Jr, A.S. and Paulino, G.H., "Convex topology optimization for hyperelastic trusses based on the ground-structure approach," Structural and Multidisciplinary Optimization, Vol. 51, No. 2, 2015, pp. 287-304. http://doi.org/10.1007/s00158-014-1147-2

[27] James, K.A. and Waisman, H., "Layout design of a bi-stable cardiovascular stent using topology optimization," Computer Methods in Applied Mechanics and Engineering, Vol. 305, 2016, pp. 869-890. https://doi.org/10.1016/j.cma.2016.02.036

[28] Kim, N.H., Introduction to nonlinear finite element analysis, Springer Science and Business Media, 2014.

[29] Belytschko, T., Liu, W.K., Moran, B. and Elkhodary, K., Nonlinear finite elements for continua and structures, John wiley and sons.

[30] Basar, Y. and Weichert, D., Nonlinear continuum mechanics of solids: fundamental mathematical and physical concepts, Springer Science and Business Media.

[31] Batoz, J.L. and Dhatt, G., "Incremental displacement algorithms for nonlinear problems," International Journal for Numerical Methods in Engineering, Vol. 14, No. 9, 1979, pp. 1262-1267. http://dx.doi.org/10.1002/nme.1620140811

[32] Sigmund, O. and Petersson, J., "Numerical instabilities in topology optimization: a survey on procedures dealing with checkerboards, mesh-dependencies and local minima," Structural optimization, Vol. 16, No. 1, 1998, pp. 68-75. http://doi.org/10.1007/BF01214002

[33] Jog, C.S. and Haber, R.B., "Stability of finite element models for distributed-parameter optimization and topology design," Computer methods in applied mechanics and engineering, Vol. 130, No. 3-4, 1996, pp. 203-226. http://doi.org/10.1016/0045-7825(95)00928-0

[34] Sigmund, O., "On the usefulness of non-gradient approaches in topology optimization," Structural and Multidisciplinary Optimization, Vol. 43, No. 5, 2011, pp. 589-596. http://doi.org/10.1007/s00158-011-0638-7

[35] Svanberg, K., “The method of moving asymptotes-a new method for structural optimization," International journal for numerical methods in engineering, Vol. 24, No. 2, 1987, pp. 359-373. http://dx.doi.org/10.1002/nme.1620240207

[36] Karush, W., "Minima of functions of several variables with inequalities as side conditions," Doctoral dissertation, 1939. 
[37] Kuhn, H.W., "Nonlinear programming: a historical view," Traces and Emergence of Nonlinear Programming, Springer Basel, 2014, pp. 393-414.

http://dx.doi.org/10.1007/978-3-0348-0439-4_18

[38] Allaire, G. and Francfort, G.A., " A numerical algorithm for topology and shape optimization," Topology design of structures, Vol. 227, NATO ASI Series, 1993, pp. 239-248.

[39] Bathe, K.J., Ramm, E. and Wilson, E.L., " Finite element formulations for large deformation dynamic analysis," International Journal for Numerical Methods in Engineering, Vol. 9, No. 2, 1975, pp. 353-386.

http://dx.doi.org/10.1002/nme.1620090207 